\title{
Investigation of Degradative Signals on Outdoor Solid Insulators Using Continuous Wavelet Transform
}

\author{
Cengiz Polat Uzunoglu ${ }^{\dagger}$
}

\begin{abstract}
Most outdoor solid insulators may suffer from surface degradations due to non-stationary currents that flow on the insulator surface. These currents may be classified as leakage, discharge and tracking currents due to their disturbing potencies respectively. The magnitude of these currents depends on the degree of the contamination of surface. The leakage signals are followed by discharge signals and tracking signals which are capable of forming carbonized tracking paths on the surface between high voltage and earth contacts (surface tracking). Surface tracking is one of the most breakdown mechanisms observed on the solid insulators, especially polymers which may cause severely reduced lifetime. In this study the degradations observed on polyester resin based insulators are investigated according to the IEC 587 Inclined Plane Test Standard. The signals are monitored and recorded during tests until surface tracking initiated. In order to prevent total breakdown of an insulator, early detection of tracking signals is vital. Continuous Wavelet Transform (CWT) is proposed for classification of signals and their energy levels observed on the surface. The application of CWT for processing and classification of the surface signals which are prone to display high frequency oscillations can facilitate real time monitoring of the system for diagnosis.
\end{abstract}

Keywords: Continuous wavelet transform, Breakdown, Solid insulator, Tracking, Discharge, Leakage

\section{Introduction}

High voltage (HV) polymeric insulators are frequently used in outdoor installations of power systems, because of their satisfying insulation properties such as light weight, higher mechanical strength and better performance in presence of heavy pollution in wet conditions [1]. Due to non-healing characteristics of solid insulators, breakdown of an insulator is irreversible and hence total breakdown may occur. On-line monitoring systems may detect total power system failure originated on a single insulator, but it is not expected to save insulator itself when breakdown initiates [2].

Some studies are being conducted to enhance the reliability and the performance of insulation materials [34]. The non-stationary currents may lead to continuous discharges and oscillations on the surface of an insulator, followed with hot spots and dry bands which finally end up as carbonized tracking paths (total breakdown) [5]. The lifetime of an insulator can be increased by cleaning up its contaminated surface which might limit surface discharges before a total breakdown occurs.

In this study polyester resin based insulators are investigated according to the IEC 587 Inclined Plane Test Standard [6-7]. During these tests surface condition of the insulators are observed and current signals are recorded.

\footnotetext{
$\dagger$ Corresponding Author: Electrical \& Electronics Engineering Department, Istanbul University, Istanbul, Turkey. (polat@istanbul.edu.tr)

Received: June 1, 2015; Accepted: November 9, 2015
}

Leakage current exhibits pure sinusoidal waveform whereas discharge and tracking signals depicts oscillations and shoulders on the sinusoidal wave [2, 5]. It is considerably an easy task to determine leakage currents on the insulator; however it is much more complicated to classify current signals either as discharge or as tracking.

There are some ongoing studies presented about determination surface condition by monitoring leakage signals [8-11]. Surface tracking leads to total breakdown which is initiated by tracking currents which are highly disturbing. On the other hand, temporary discharge signals don't lead to breakdown of an insulator; however they needed to be monitored continuously against the probability of transforming into tracking signals. The primary goal of this study is to identify the surface condition of an insulator by classifying current signals in terms of discharge or tracking characteristics, since only the tracking signals can be resulted in breakdown of an insulator.

In literature, some methodologies were developed for monitoring surface conditions by using neural network based data analysis. These methods are basically based on the extracted features of Fast Fourier Transform (FFT) of surface current signals $[2,11]$. FFT analysis is a quite satisfactory tool for stationary signals, however for nonstationary signals such as discharge and tracking signals, FFT analysis cannot reveal information about particular frequency rises on the signal. Non-stationary signals depict varying time statistics and cannot be handled as deterministic or stationary signals. 
It is quite possible to confuse discharge and tracking signal by using well-known methods such as FFT and time domain analysis. In this study due sharp spikes and discontinuities of tracking and discharge signals, CWT technique [12] is proposed for determination of tracking signals. Wavelets are mathematical functions which initially slice data into different frequency components and then focus on each component with a resolution matched to its scale [13]. Instantaneous energy signals of surface currents exhibits distinctive characteristics, which are useful for interpreting tracking signals. In this work real time monitoring of surface condition with CWT enables continuous detection of surface signals and instantaneous energy signals is proposed.

\section{Test Set-up}

In this study, tracking phenomenon in polyester resin based test samples has been studied under AC voltage, with ammonium chloride $(\mathrm{NH} 4 \mathrm{Cl})$ as a contaminant. All test specimens are tested according to the IEC 587 Inclined Plane Test standard [6]. The test set-up is given in Fig. 1.

The HV and earth electrodes are fixed on the test sample with the dimension of $120 \times 50 \times 6 \mathrm{~mm}$. The sample is mounted at $45^{\circ}$ to the horizontal and the test voltage is set to $4 \mathrm{kV}$. The upper part of the test specimen is connected to the $\mathrm{HV}$ electrode and the lower part of the specimen is connected to ground electrode through a series $100 \Omega$ resistor. The test signals are monitored by measuring the voltage across this resistor. The buffer unit is connected for eliminating over voltages for protection of measurement system. The current signals are monitored by scope and recorded to a personal computer through an A/D converter.

According to the standard test, distilled water with the contaminant of $0.1+0.002 \%$ ammonium chloride with a flow rate of $36 \mathrm{ml} / \mathrm{h}$ is supplied $[2,6]$. During the tests surface of the test specimen is constantly monitored and when the tracking initiation is observed the current waveform is recorded and labeled as tracking signal. During the test period all non-tracking signals are labelled as discharge and leakage signals depending on their waveform characteristics.

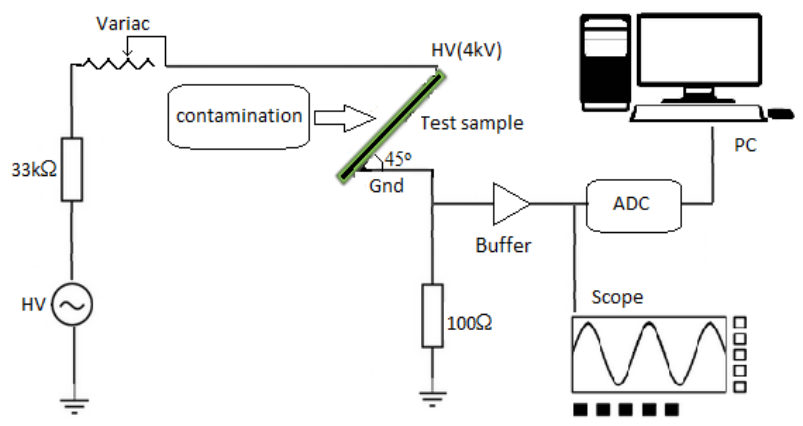

Fig. 1. The test set-up

\section{Signal Model}

The surface conditions of the solid insulators are strictly related with the current waveforms observed on the insulator. The standard test equipment is operated at $4 \mathrm{kV}$ $50 \mathrm{~Hz}$ voltage. The observation signal obtained from test sample can be described with single sinusoid and an additive noise which is given in Eq. 1 .

$$
z=x+v
$$

Also an observation signal $\mathrm{z}$, which is measured on the test sample can be denoted as a sum of a sinusoid $\mathrm{x}$ with additive noise $\mathrm{v}$.

$$
\begin{gathered}
x_{k}=a \cos \left(w t_{k}+\Phi\right) \\
w=2 \pi f
\end{gathered}
$$

in which a, f and $\Phi$ are amplitude, frequency and the phase of the sinusoid (observation signal) respectively. The sample leakage current and notch filtered $(50 \mathrm{~Hz})$ [14] leakage noise measured from test set-up is given in Fig. 2. Approximately two and a half period (0.05 s) of observation signal is taken into consideration.

The leakage noise can be modelled as low level Gaussian white noise with zero-mean and variance. As seen from Fig. 2 leakage signal is assumed to be a stationary signal. So it is easy to rule out leakage signal due to non-degradative properties (lack of oscillations) of the leakage currents. On the other hand discharge signal exhibits high level peaks and oscillations as well, which are given in Fig. 3.

The discharge noise contains sharp peaks and oscillations which states non-stationary signal characteristics. These sharp peaks are approximately 0.4 p.u. strength of the fundamental test signal and have considerable time differences between them. Rest of the noise displays Gaussian noise characteristics. The tracking signal which is
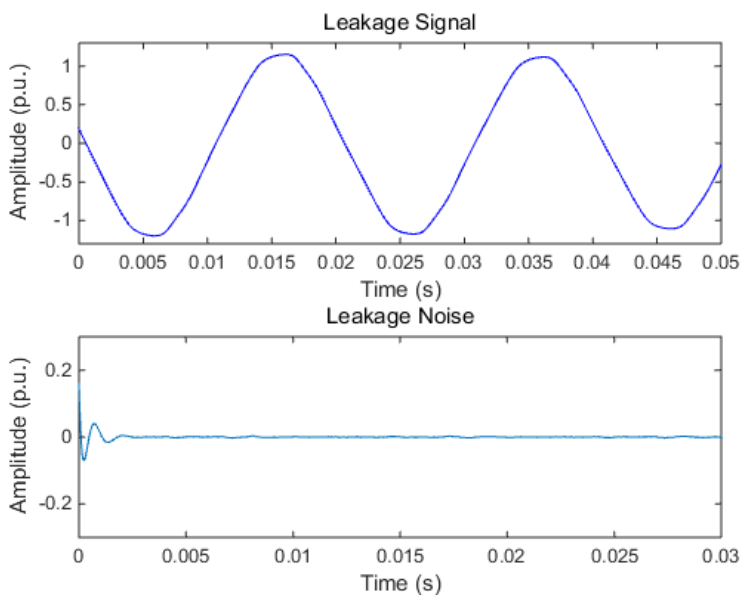

Fig. 2. The leakage current and leakage noise 

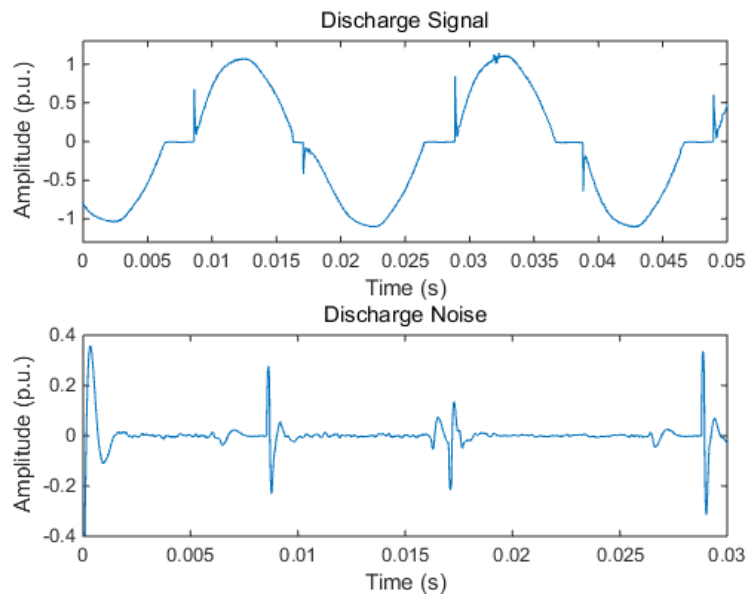

Fig. 3. The discharge current and discharge noise
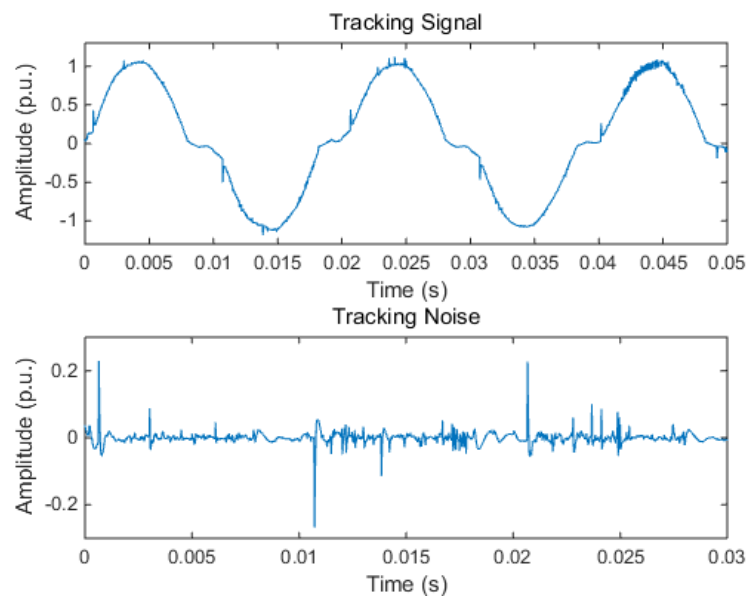

Fig. 4. The tracking current and tracking noise

recorded after tracking initiation and also tracking noise are given in Fig. 4. Tracking signals exhibit continuous oscillations and limited number of peaks. These peaks are less powerful than the ones observed with the discharge signal; however tracking signal has repeatedly small scale oscillations all test time period. These continuous degradative characteristics of this signal type leads to tracking initiation on the insulator surface and represents final phase before total breakdown.

FTT analysis of a discharge and tracking signal are given in Fig. 5. Due to the non-stationary characteristics of discharge and tracking signals, FFT can reveal frequency components considering the whole of the time signal [2].

However, the tracking phenomenon idea lays on the small frequency fragments, such as peaks and oscillations. For this purpose instantaneous energy levels of the surface signals are used.

\subsection{Instantaneous energy}

The instantaneous energy (in joule) of a time domain signal, which is sampled and recorded by digital oscilloscope, is given in Eq. 4.
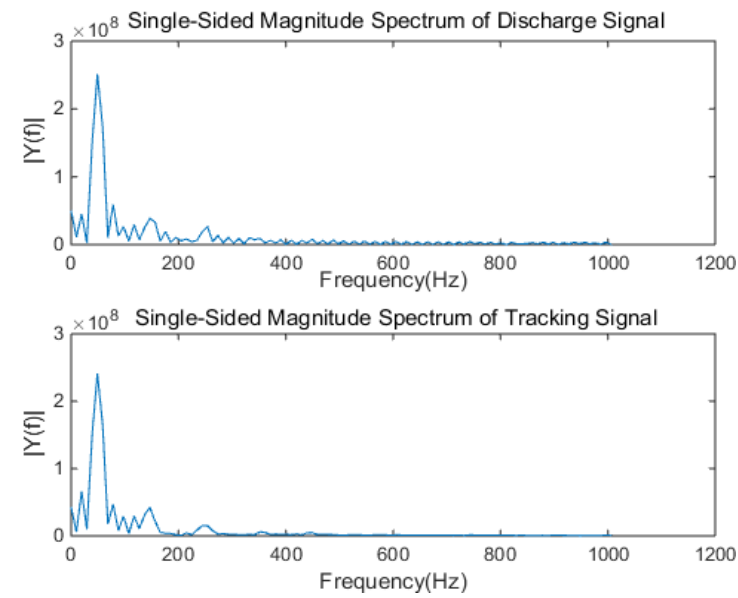

Fig. 5. FFT of discharge and tracking signals
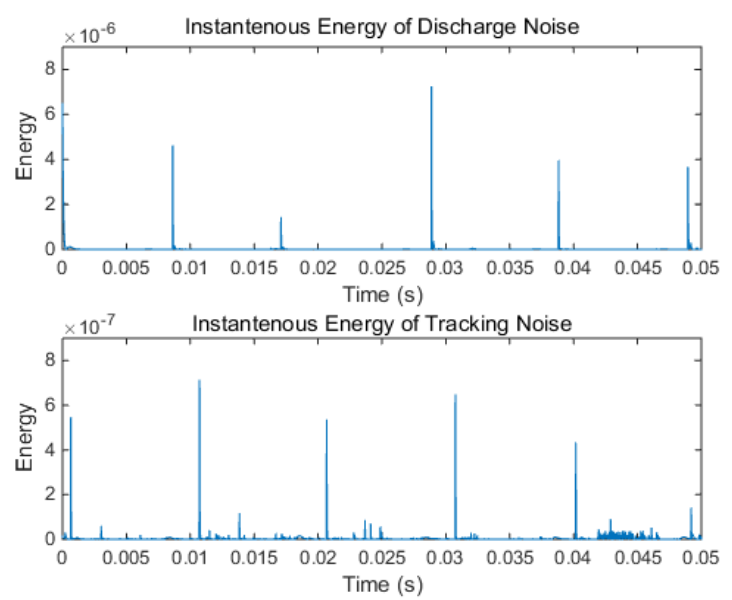

Fig. 6. Instantaneous energy levels of discharge and tracking signals

$$
W_{n}=\frac{U_{n}^{2}}{Z f_{s}}
$$

In the expression, $U_{n}$ represents sampled signal amplitude, $Z$ represents input impedance of the proposed measurement system and $f_{s}$ represents sampling frequency. $W_{n}$ is the instantaneous energy level of a signal which is calculated for every sample in time domain. Also it is possible to calculate total energy sequence of the observation signal by using Eq. 5 .

$$
E_{n}=W_{n}+E_{n-1}, \quad n=1,2, \ldots ., N-1, N
$$

In this study, in order to obtain more accurate classification results of surface conditions the instantaneous energy levels of time signals are calculated. The instantaneous energy levels of the surface signals are given in Fig. 6.

The energy peaks of a discharge signal are distributed discretely over time as expected, whereas the energy pattern of tracking signals is distributed continuously over time. The tracking initiation is observed during constant energy 
spikes over the insulator surface. Further investigations of the surface signals are needed for a proper classification of tracking signals on a real time basis, hence for this purpose CWT method is proposed.

\section{Continuous Wavelet Transform (CWT)}

Wavelet analysis has advantages over traditional Fourier transform techniques in terms of physical variations such as discontinuities, sharp transitions and spikes. Wavelet has numerous applications including transient signal analysis, mechanics, communications, radar, pattern recognition etc. [15-18]. The wavelet transform converts a signal by using small wavelets (wave-like oscillation) from time domain to the time-scale domain within limited time duration [12]. Wavelet transform is based on a single specific function which is called mother wavelet. The transformation is conducted by reforming original signal by using shifted and scaled versions of mother wavelet functions. The $\psi(\mathrm{t})$ is an complex-valued mother wavelet function (or signal) defined in a vector space for finite energy signals and such complex-valued signals (in general) satisfy;

$$
\int_{-\infty}^{\infty} \psi^{2}(t) d t<\infty
$$

This condition satisfies finite energy of this function. The second condition which is given in Eq. 7 satisfies the smoothness of the function in frequency domain, while Fourier Transform of this function for zero frequency is $\operatorname{FFT} \Psi(0)=0$. These functions are called mother wavelet functions $[12,15]$.

$$
C \psi=2 \pi \int_{-\infty}^{\infty} \frac{|F F T \Psi|^{2}}{|\omega|} d \omega<\infty
$$

The wavelet transform of a given real signal $(\mathrm{z}(\mathrm{t}))$ can be obtained with respect to the mother wavelet function:

$$
C W T_{z}^{\psi}(\tau, s)=\frac{1}{\sqrt{|s|}} \int z(t) \psi^{*}\left(\frac{t-\tau}{s}\right)
$$

where $\psi^{*}$ denotes complex conjugate of mother wavelet function (base function) and transformation lay on the plane ( $\tau, \mathrm{s} \in \mathrm{R}$ and $\mathrm{s}>0$ ) which is defined by $\tau$ and $\mathrm{s}$ parameters. The $\tau$ parameter is called dilation parameter and it corresponds to time-shift. Also the s parameter is called translation parameter and the scaling factor of the wavelet transform is defined by this parameter [19]. In this study due to quick response time and high frequency characteristics during instantaneous oscillations of observation signal, a Meyer-type wavelet transformation is proposed [20-22].

\section{Experimental Results}

During the test period, surface condition of the test sample is monitored and the corresponding signal waveforms are recorded simultaneously. In order to achieve a proper classification of tracking signals in real time basis, CWT of time and energy signals is analyzed. Ten different observation signals for each signal waveforms are recorded and processed. The CWT's of discharge and leakage signals are given in Fig. 7.

The main tracking source contains repeated oscillations and peaks on the surface current. The time differences of repeated oscillations define the frequency of the oscillations. The time differences of consecutive oscillations and the number of oscillations are measured by signal processing techniques. Real time detection of surface tracking signals is established by using combination of CWT and peak measurement. The number of consecutive oscillations and corresponding time differences for CWT of surface signals are given in Fig. 8.
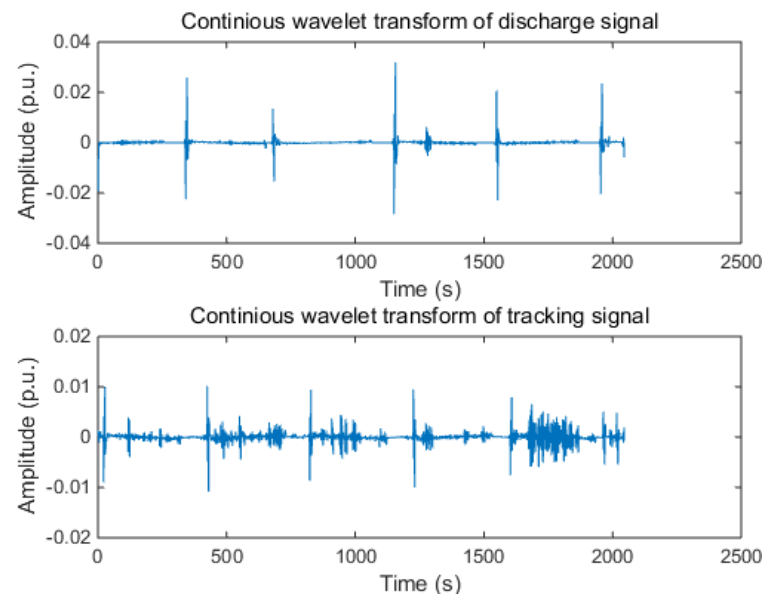

Fig. 7. CWT of discharge and tracking signals
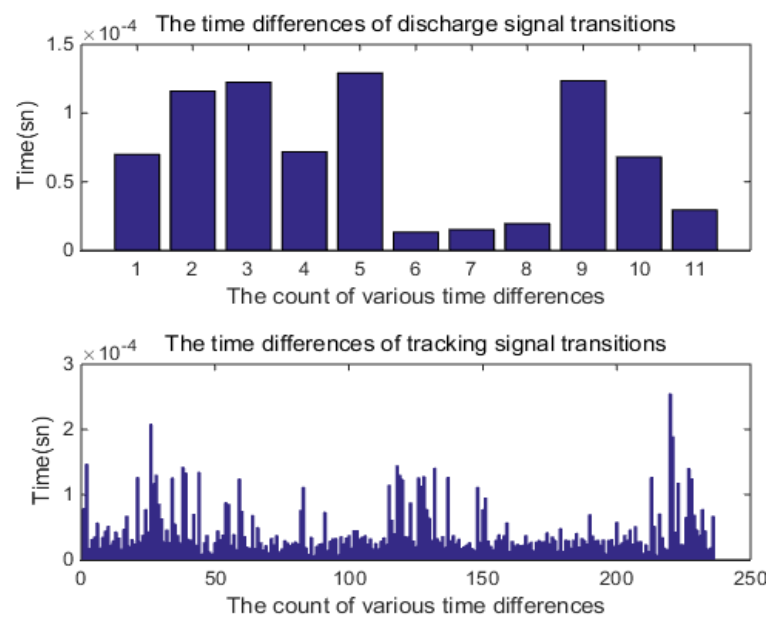

Fig. 8. The time differences of consecutive oscillations on CWT of surface signals 
Table 1. The CWT statistics of discharge and tracking signals

\begin{tabular}{c|c|c}
\hline Waveform Type & Discharge & Tracking \\
\hline Total time & $51.2 \mathrm{~ms}$ & $51.2 \mathrm{~ms}$ \\
\hline Oscillation number & 11 & 236 \\
\hline Minimum time difference & $\begin{array}{c}13.1 \mu \mathrm{s} \\
(2.5 \% \text { of total })\end{array}$ & $\begin{array}{c}6.05 \mu \mathrm{s} \\
(1.2 \% \text { total })\end{array}$ \\
\hline Maximum time difference & $0.129 \mathrm{~ms}$ & $0.25 \mathrm{~ms}$ \\
\hline Average of time difference & $4.65 \mathrm{~ms}$ & $216 \mu \mathrm{s}$ \\
\hline
\end{tabular}
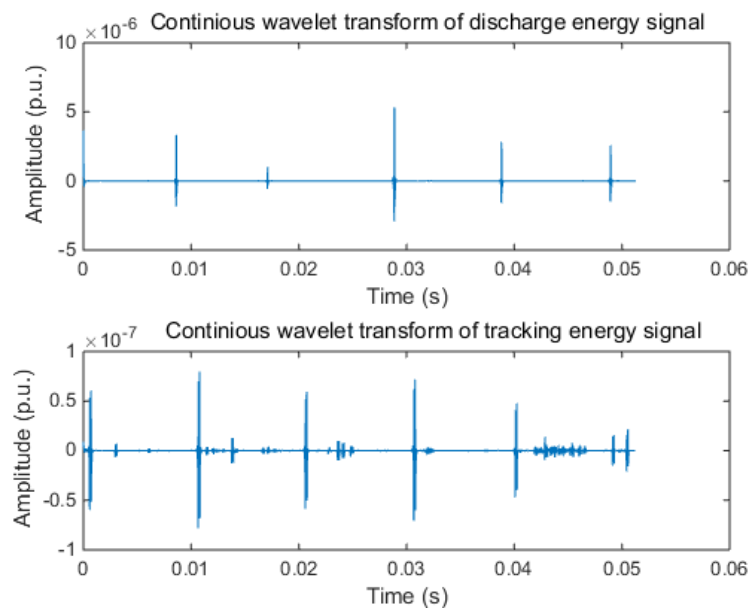

Fig. 9. The CWT of discharge and tracking energy signals

The time statistics for wavelet transforms of time signals are given in Table 1. The oscillation numbers, the minimum time differences of consecutive oscillations, the maximum time differences of consecutive oscillations and average of time differences are provided. The main difference between discharge and tracking signal is how frequently oscillations and peaks are repeated over a specific time period. The tracking signals contain much more oscillations and less time difference of consecutive oscillations as well.

After analyzing the data, obtained by average statistics over ten different samples of each kind of waveform, CWT of instantaneous energy signals depict much more accurate results compared to the CWT of time signals. The CWT's of discharge and leakage energy signals are given in Fig. 9.

The CWT of energy signals states instantaneous transitions and oscillations in a distinct way hence, by using these signals it is a much easier task to distinguish tracking signals. The number of consecutive oscillations and corresponding time differences for CWT of energy signals are given in Fig. 10. The CWT of energy signals displays more distinctive properties than the ones provided by CWT of time signals (Fig. 7). The frequency of various oscillations of tracking signals is extremely high; on the other hand, the frequency of various oscillations of discharge signals is very low.

The time statistics for wavelet transforms of energy signals are given in Table 2. The oscillation numbers of tracking signals are increased and time differences between two consecutive oscillations are reduced in contrast to CWT analysis of regular signals. Tracking initiation time
Table 2. The CWT statistics of discharge and tracking energy signals

\begin{tabular}{c|c|c}
\hline Waveform Type & Discharge & Tracking \\
\hline Total time & $51.2 \mathrm{~ms}$ & $51.2 \mathrm{~ms}$ \\
\hline Oscillation number & 3 & 554 \\
\hline Minimum time difference & $\begin{array}{c}9.9 \mathrm{~ms} \\
(19 \% \text { of total })\end{array}$ & $\begin{array}{c}0.103 \mu \mathrm{s} \\
(0.02 \% \text { total })\end{array}$ \\
\hline Maximum time difference & $20.2 \mathrm{~ms}$ & $0.213 \mathrm{~ms}$ \\
\hline Average of time difference & $13.4 \mathrm{~ms}$ & $32.3 \mu \mathrm{s}$ \\
\hline
\end{tabular}
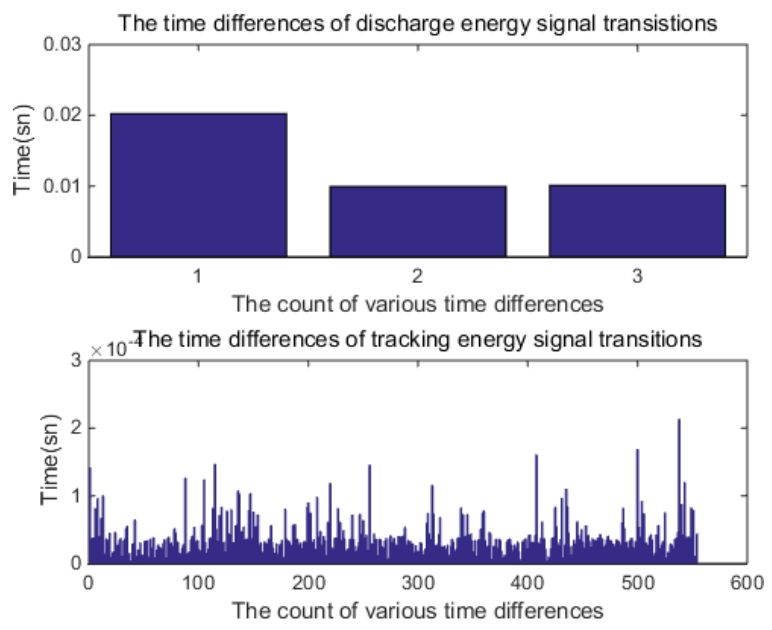

Fig.10. The time differences of consecutive oscillations on CWT of energy signals

can be estimated by measuring oscillation numbers and corresponding time differences of CWT results.

\section{Conclusion}

In this study, investigations of the surface conditions and breakdown possibility on the polymeric insulator surfaces are conducted according to IEC 587 Inclined Plane Tracking Test by using CWTs of surface currents. Surface discharges and degradations are possible consequences when the solid insulator is exposed to pollution conditions. During these tests it is observed that the leakage and discharge currents are not capable of initiating breakdown. However due to the non-stationary characteristics of tracking and discharge signals, it is not easy task to determine tracking signal, which is highly degradative, by using conventional FFT method or time domain analysis. The main idea of signal classification depends on measuring how frequent oscillations or peaks are occurred on the insulator surface. Unlike the FFT analysis, CWT can focus on limited time duration, which is desired property for non-stationary signals especially containing repeated oscillations. Since the instantaneous energy signals of surface currents are much more distinctive, CWT of energy signals are proposed and better classification results are obtained. The CWT technique can be adapted 
for automation system which can identify the surface conditions depending on the surface current waveforms in operation, accurately and hence the proposed method may come up with better design ideas of insulators especially for certain insulation points. This method can also utilize on-line detection of insulation structure in a power system for possible inception of surface degradation which satisfies the entire system reliability.

\section{Acknowledgements}

This work was supported by Istanbul University Research Fund with the project code 56920. I would like to thank Istanbul University Research Fund for this financial support.

\section{References}

[1] R. Hackam, "Outdoor HV composite polymeric insulators," Dielectrics and Electrical Insulation, IEEE Transactions on, vol. 6, no. 5, pp. 557-585, 1999.

[2] M. Ugur, D. W. Auckland, B. R. Varlow, Z. Emin, "Neural networks to analyze surface tracking on solid Insulators," IEEE Transactions on Dielectrics Electrical Insulation, vol.4, no.6, pp.763-766, 1997.

[3] J. W. Kim, Y. S. Roh, "Simulation on Surface Tracking Pattern Using the Dielectric Breakdown Model," Journal of Electrical Engineering \& Technology, vol.6, no.3, pp.391-396, 2011.

[4] K. Y. Lau, M. A. M. Piah, "Polymer nanocomposites in high voltage electrical insulation perspective: a review," Malaysian Polymer Journal, vol. 6, no. 1, pp. 58-69, 2011.

[5] V. Rajini, K. Kanchana, V. Gowrishree, K. Udayakumar, "Comparison of surface tracking in polymeric insulating materials," PowerCon 2004, vol. 2, pp. 1513-1517, 2004.

[6] IEC 587, "Test Methods for Evaluating Resistance to Tracking and Erosion of Electrical Insulating Materials used Under Severe Ambient Conditions," 1986.

[7] M. Afendi, M. Piah, A. Darus, T. Tamsir, "Mathematical analysis for parameters variation in inclinedplane tracking test method," TENCON '02 IEEE Region 10 Conference on Computers, vol. 3, pp. 1877-1880, 2002.

[8] C. Muniraj, S. Chandrasekar, "Analysis of leakage current on polluted polymer insulator by high resolution spectrum estimation method," ICPS '09 International Conference on, pp. 1-5, 2009.

[9] Z. Yong, K. Haji, H. Yamamoto, T. Miyake, M. Otsubo, C. Honda, "Distribution of leakage current on polluted polymer insulator surface," Electrical Insulation and Dielectric Phenomena, 2006 IEEE
Conference on, pp.397-400, 2006.

[10] M. Afendi, M. Piah, A. Darus, "Leakage current analysis of polymer insulating material with variable contaminant flow rate," Australasian Universities Power Engineering Conference, Melbourne, Australia, 2002.

[11] S. Kumagai, N. Yoshimura, "Leakage current analysis for monitoring the conditions of polymer insulators," Electrical Insulating Materials, Proceedings of 2001 International Symposium on, pp.55-58, 2001.

[12] S. M. H. Hosseini, P. R. Baravati, "Partial Discharge Localization Based on Detailed Models of Transformer and Wavelet Transform Techniques," Journal of Electrical Engineering \& Technology, vol.10, no.3, pp.1019-1101, 2015.

[13] A. Graps, "An introduction to wavelets," Computational Science \& Engineering, IEEE, vol. 2, no. 2, pp. 50-61, 1995.

[14] M. Mojiri, M. Karimi-Ghartemani, A. Bakhshai, "Time-domain signal analysis using adaptive notch filter," Signal Processing, IEEE Transactions on, vol. 55, no. 1, pp. 85-93, 2007.

[15] D. T. Lee, A. Yamamoto, "Wavelet analysis: theory and applications," Hewlett Packard Journal, vol. 45, pp. 44-44, 1994.

[16] M. J. Medley, G. J. Saulnier, P. K. Das, “Applications of the wavelet transform in spread spectrum communications systems," In SPIE's International Symposium on Optical Engineering and Photonics in Aerospace Sensing, pp. 54-68, 1994.

[17] Y. S. Oh, J. Han, G. H. Gwon, D. U. Kim, C. H. Kim, "Development of Fault Detector for Series Arc Fault in Low Voltage DC Distribution System Using Wavelet Singular Value Decomposition and State Diagram," Journal of Electrical Engineering \& Technology, vol.10, no.3, pp.766-776, 2015.

[18] M. Rucka, K. Wilde, "Application of continuous wavelet transform in vibration based damage detection method for beams and plates," Journal of Sound and Vibration, vol. 297, no. 3-5, pp. 536-550, 2006.

[19] A. Cohen, J. Kovacevic, "Wavelets: The mathematical background," Proc. IEEE, vol. 84, no. 4, pp. 514-522. 1996.

[20] Y. Meyer, "Wavelets-algorithms and applications," Wavelets-Algorithms and applications Society for Industrial and Applied Mathematics Translation, vol. 142, pp. 1, 1993.

[21] I. Daubechies, "Ten lectures on wavelets," Philadelphia: Society for industrial and applied mathematics, vol. 61, 1992.

[22] X. Zhang, C. Deng, Y. Han, "The image space of meyer wavelet transform," Measurement, Information and Control (ICMIC), 2013 International Conference on, vol. 02, pp. 1136-1139, 16-18 Aug. 2013. 


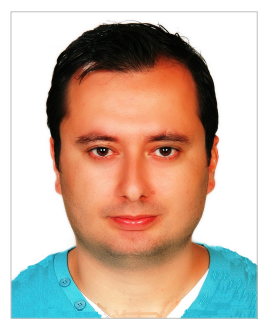

Cengiz Polat Uzunoglu received the M.Sc. and Ph.D. degrees in Electrical Electronics Engineering from Istanbul University, Istanbul, Turkey, in 2005 and 2011, respectively. Since 2003, he has been working as research assistant in the Department of Electrical and Electronics Engineering of Istanbul University. His main research interests include power systems, chaotic systems, high voltage engineering and non-linear systems. 